\title{
A Smart Walking Stick Powered by Artificial Intelligence for the Visually Impaired
}

\author{
Siddhi Degaonkar
PES Modern College of \\ Siddhi Degaonkar
PES Modern College of \\ Engineering
}

\author{
Musahib Singh \\ PES Modern College of Engineering
}

\author{
Mohit Gupta \\ PES Modern College of \\ Engineering
}

\author{
Prajakta Hiwarkar \\ PES Modern College of \\ Engineering
}

\author{
S. A. Itkar, PhD \\ PES Modern College of Engineering
}

\begin{abstract}
The purpose of developing a cost-effective smart walking stick for the visually impaired and the need increased with a steady increase in their population worldwide. The smart stick presented in this paper uses artificial intelligence along with ultrasonic sensors to help the visually disabled people to navigate through the environment independently. Image recognition, Collision detection and obstacle detection are the tasks performed on a raspberry pi having high processing power. The task of obstacle detection and obstacle avoidance makes use of ultrasonic sensors to alert the user of the obstacles appearing in his path. The smart walking stick also demonstrated the important characteristics of affordability, mobility and high performance
\end{abstract}

\section{Keywords}

Visually impaired, Image Recognition, Collision Detection, Artificial Intelligence, Ultrasonic Sensors, Raspberry Pi.

\section{INTRODUCTION}

According to the estimates of World Health, Organization, about 45 to 50 million people are blind [1]. With the staggering number of visually disabled people around the world, providing care for such a large population results in an enormous socioeconomic stress on the countries. About $90 \%$ of global visually impaired people belong to low-income households with highest numbers in developing countries like India $[1,2]$. This trend will continue to grow because of widespread chronic eye diseases, population growth as well as the increase in the number of recently born disabled children which can be because of a genetic disorder or natural birth defects.[2]. People with visual disability have a constant need of assistance in their daily lives. It can range from relying on other people for help to using various Electronic Travel Aid assistive devices whenever needed. People also use Guide dogs and white canes but the restriction on the allowance of guide dogs in certain places and short range of white canes serve as their shortcomings.

Similarly most of the ETAs also have drawbacks of being unaffordable and inefficient. Keeping a tab on the various drawbacks of traditional and common methods of providing assistance to the blind and visually impaired people, we decided to develop and design our system with a novel approach. Past few years have seen wide scale applications of artificial intelligence ranging from national defence, scientific research, automated vehicles to space exploration. Taking advantage of the monumental advances in artificial intelligence and helping visually impaired people was our main goal. For the features we desired and the tasks which we intended our system to perform, depending solely on artificial intelligence was not feasible. So using a microcontroller and sensors based hardware in addition to artificial intelligence was decided upon. The system thus not only makes use of artificial intelligence capabilities but also gathers precise readings from the various sensors to become a real-time assistive system. This enables the visually impaired people to navigate the world independently with confidence.

\section{RELATED WORK}

A significant amount of research has been published previously to develop an effective system for the visually impaired and blind people. One such work described bringing the modules for Image Recognition, Obstacle Detection and Obstacle avoidance to work together. The drawbacks of this system was that the sensors were mounted on the stick and image processing was done on a mobile application making the system bulky and also increasing the time required to return the output to the user. Another system that was studied for this paper was The Artificial Vision System for Blind [3], consisted of ultrasonic sensors and a microcontroller that returned the distance of the obstacles in the vicinity of the user and to suggest him towards an alternative route via an audio output. The drawback of this system was that it blocked hearing of the visually impaired individuals. The limitations of having a heavy prototype and use of audio feedback again blocking the hearing ability [4]. The Navbelt system [5] used 8 ultrasonic sensors to create a map of angle and distances of objects around the user.

All the work mentioned before not only lacked the use of new AI cloud based systems and API's but also lacked the important characteristics of mobility and affordability. Focus on implementing obstacle detection, collision detection and image recognition together on the same prototype without adding much bulk and with an independent power source with a good output to keep the system functioning for some hours was also absent. Learning from the drawbacks and building upon the positives of these earlier works helped us design a better system. We presented a brief explanation of our intellect of developing a cost-effective and mobile smart walking stick for the visually disabled people. The implementation of image processing techniques for edge detection and texture detection and background dimming for image recognition purposes often ended with certain error-prone results. The hardware implementation also required numerous iterations of fine tuning and the software implementation required algorithms with a fine sense of precision to make a prototype work seamlessly. 


\section{PROPOSED SYSTEM}

The smart stick was developed to leverage the drawbacks of other models and consists of a 3.5-4 foot long stick. The stick consisting of a Raspberry Pi v3 B+ microcontroller, an ultrasonic sensor, web camera or a pi camera and a vibrating motor module as shown in figure 2 . The power source to this stick is a massive $10000 \mathrm{MaH}$ power bank which provides ample battery backup powering on the stick, the user starts walking by holding the stick in hand. The raspberry pi and the sensors on the stick then start a recursive analysis of the environment in the user's path. In order to get some idea of his surroundings, the pi/webcam captures images and in every interval of 15 seconds. These images are then processed by the Artificial intelligence module in the Raspberry Pi leading to tag generation with confidence levels after which caption is generated for each image which describes their contents. These captions are then sent to the user returning them a voice output through its earphones. The ultrasonic sensor mounted on the front side of the stick detects the approximate distance of the obstacles nearing in front of the user and an audio feedback and varying intensity vibrations are provided to the user so that he alters his route to avoid colliding with the obstacles. The flowchart of the proposed prototype is as shown below.

The Raspberry Pi sends these values to the user by either activating the vibration motor or returning an alert in the ear or both actions at the same time. The HC-SR04 ultrasonic sensors based on the principle of deflection of sound are tasked with identifying the recurring obstacles coming in the user's route by sending and receiving ultrasonic waves. The camera mounted on the stick clicks clear wide angle images for better results. The Raspberry $\mathrm{Pi}$ needs an internet connection for image processing, This internet connection is given by creating a secured wifi hotspot on the users smartphone. The wifi module is already present on the raspberry pi microcontroller and hence establishing a connection is convenient and safe. The computed distance values from the Raspberry Pi board are sent to the user's headphones. It also provides audio and vibrations as a feedback to the user.

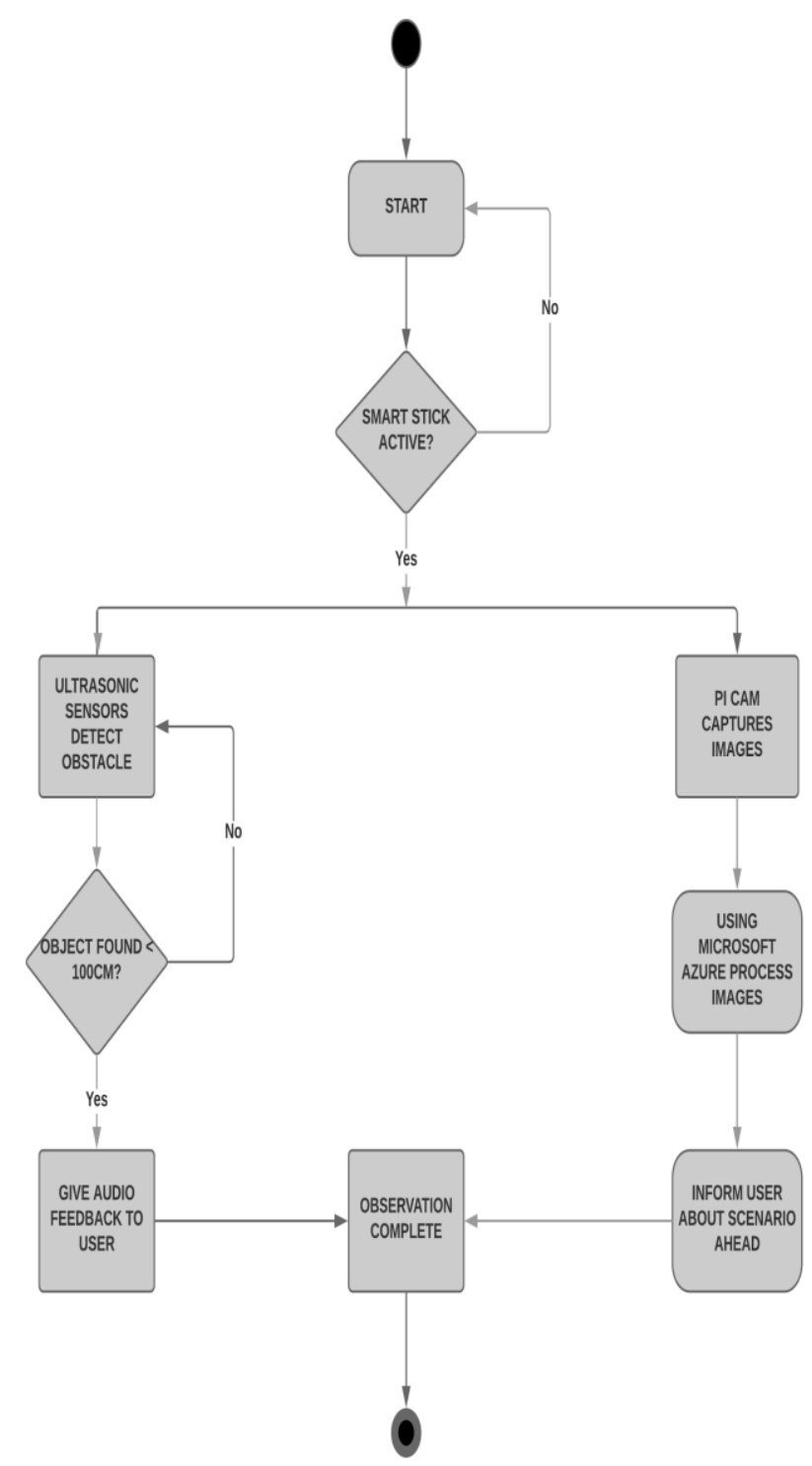

Fig. 1. Flowchart of the system

\section{IMPLEMENTATION AND RESULTS}

The smart stick performs three different tasks i.e. image recognition, collision detection and obstacle detection. Image recognition helps the visually disabled user to listen to a summarized one line description of his surroundings. Collision detection focuses on instructing the user of avoiding possible collision with an obstacle upfront in his route. Obstacle detection identifies potholes and bumps on the ground in user's route, guiding the user to avoid it. These three tasks are explained as follows: 


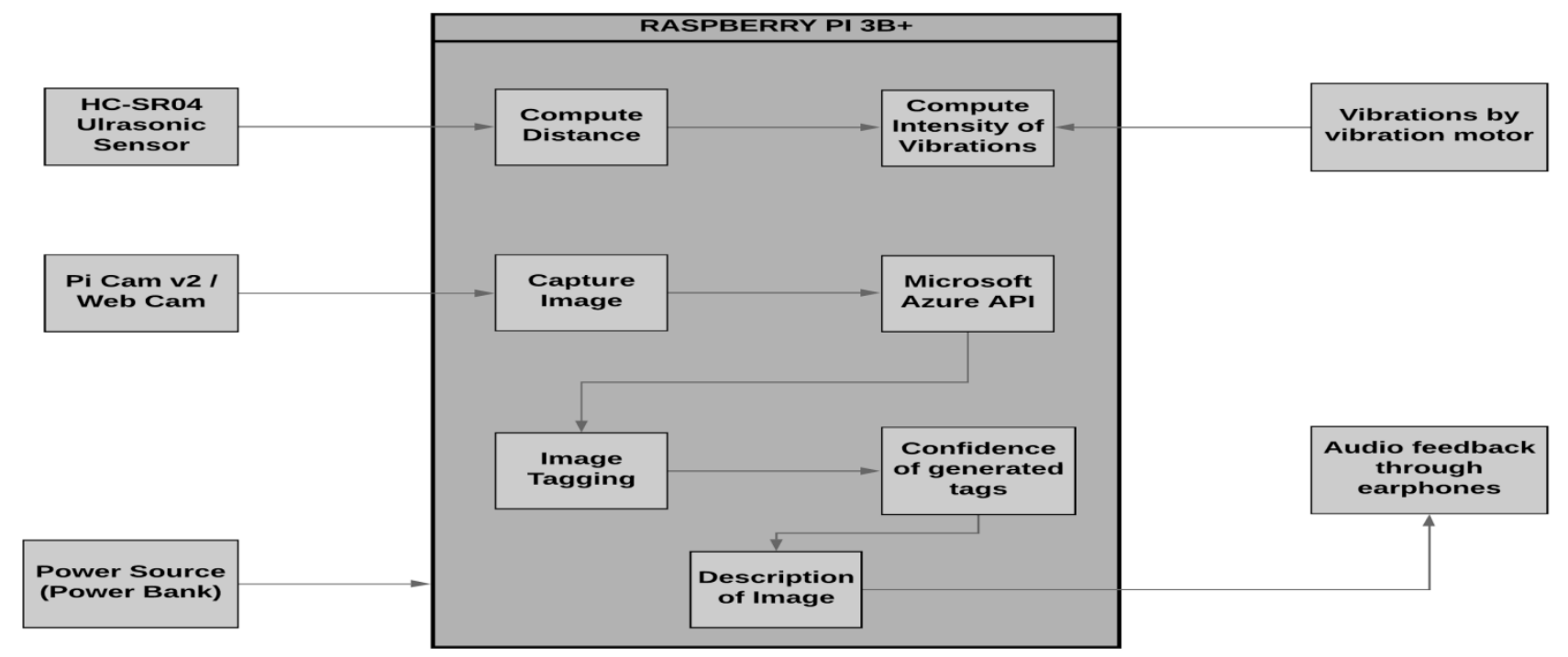

Fig. 2. Block diagram of the system

\subsection{Image Recognition}

Image description deals with extracting information from the user captured images and narrate this information back to the user. For this purpose, the user holds the pi camera in front of him and the raspberry pi captures. The images are then described by the application's artificial intelligence eventually generating captions for every user captured image. Google TalkBack identifies the generated captions and speaks it to the user via the earphones connected to the raspberry pi [6]. This allows the user to get a good perception of his surrounding environment and helps him to better navigate his route.The Raspberry Pi uses a mobile hotspot to perform artificial intelligence related tasks. The Computer Vision API of Microsoft Cognitive Services is what really powers the artificial intelligence in our system [7]. Whenever the need to analyze an image arises our system application code invokes a call to this API. Image analysis by the API is done using computer vision, machine learning and neural networks whereas natural language processing is used to generate the caption in a human understandable language [8]. The system can analyze an image, read texts and handwritten texts from an image, identify landmarks and celebrities and also perform video analysis in near real-time. For the image caption speaking feature, we decided to use Google TalkBack service.

piQraspberrypi: /Desktop/Final/New \$ python imagerecog.py

\{u'color': \{u'dominantColorForeground': u'Brown', u'dominantcolors': [u'Creen'], U'isBWImg': False, U'dominantColorBackground': U'Creen', U'accentcolor': U'Aa6821', u'isBwImg': False\}, u'metadata': \{u'width': 259, u'format': u'Jpeg', u'height': 194\}, u'description': \{u'captions': [\{u'text': U'a dog looking at the camera', u'confidence 9.8688149766974038\}], u'tags': [u'dog', u'animal', u'grass', u'mammal', u'brown', u'sitting', u'looking', u'tan', u'yellow', u'standing', u'mouth', u'laying', u'large u'field', u'white']\}, u'categories': [\{u'score': 0.99609375, u'name': u'animal_dog'\}] u'requestid': U'a6990948-71b8-431b-9302-9366agfca816'\}

\section{Fig. 3. Result of image recognition}

\subsection{Obstacle Detection}

The ultrasonic sensors generate high frequency sound waves and evaluate the echoes received back by the sensors. For the functioning of obstacle detection, the ultrasonic sensor mounted at the stick sends ultrasonic waves towards direction at which the stick is pointing to. The waves reflected from the obstacle in that direction are received by the ultrasonic sensor.
Using the time required by the waves to return back to the sensor, the distance value between the stick and the obstacle is calculated in the Raspberry Pi using the following formula:

Approx. speed of sound $\mathrm{c}=331.5+[0 \cdot 6 \times$ (air temperature in degrees Celsius)] at 20 degrees

$\mathrm{c}=331 \cdot 5+(0 \cdot 6 \times 20)=343 \cdot 5 \mathrm{~m} \mathrm{~s}$

$\mathrm{c}=343 \cdot 5 \times 100 / 10^{\wedge} 6 \mathrm{~cm} \mu \mathrm{S} /=0 \cdot 03435 \mathrm{~cm} \mu \mathrm{S} /$ speed of sound $=1 / \mathrm{c}=29 \cdot 1$

The time interval between ultrasonic waves sent by the sensor is in $\mu \mathrm{S}$.

distance $=($ duration $/ 2) / 29.1$

\subsection{Collision Detection}

The presence of an obstacles upfront in the user's route are identified and a warning is generated in the form of vibrations and voice in the earphones. The ultrasonic sensor, mounted on the front side of the stick is utilized for this task. A distance of 4 feet has been set as the default safe distance in front of the stick wherein if any obstacle is detected inside the safe distance, the collision detection system starts warning the user. For this task, the sensor transmits ultrasonic waves towards the front direction of the user. Once the waves reflected from the obstacle are received by the sensor, the time is returned which makes distance calculations easier. Using the time difference between the moment the ultrasonic waves were sent from and received back by the sensor along with the distance formula used in the obstacle detection algorithm, the distance between the user and the obstacle in the user's route is calculated. These calculations are performed by the Raspberry $\mathrm{Pi}$ board. The computed distance value is then sent from the Raspberry Pi board to the user by a headphone or a vibration motor. The smartphone application then processes these readings and generates a series of beeping sounds thus alerting the user and causing him to alter his current route. All these tasks from calculating the distance of obstacles from the user to generating warning sounds happen in real time so that the user can act quickly and be safely away from a potentially dangerous situation. 
1 pi@eraspberrypi; /Desktop/Final/New \$ python sensor.py

22.66545820236

32.66255509853

42.6378582716

52.63646805286

62.66509020329

72.64194715023

82.6390440464

92.62788140774

102.66578531265

112.65584933758

122.66320931911

132.64452314377

142.65020668507

152.63798093796

162.64628136158

172.64779424667

182.66304576397

192.66083776951

202.65380489826

212.64317381382

\subsection{1}

\section{Fig. 4. Result of obstacle and collision detection}

Comparative study with our model and other systems [4] shows that the performance graph with respect to time has staggering differences as shown in the graph below where we consider different network spectrums and bandwidths. The results prove that our model surpassed the time required for the final output by a heavy margin.

\section{CONCLUSION}

In this paper, The proposed system is used for assisting visually impaired in their navigation. The system performs two main tasks image recognition and obstacle detection allowing user to navigate independently. Using appropriate hardware and developing application, our system manages to overcome hurdles of visually impaired. Using just the Raspberry Pi for all the processes and other high quality hardware, our system managed to overcome the hurdle of developing an assistive system which was both efficient and affordable enough for the visually impaired people especially belonging to the low-income households. Future research work involves refining our system so that a more hands free assistive system experience can be provided for the visually disabled people.

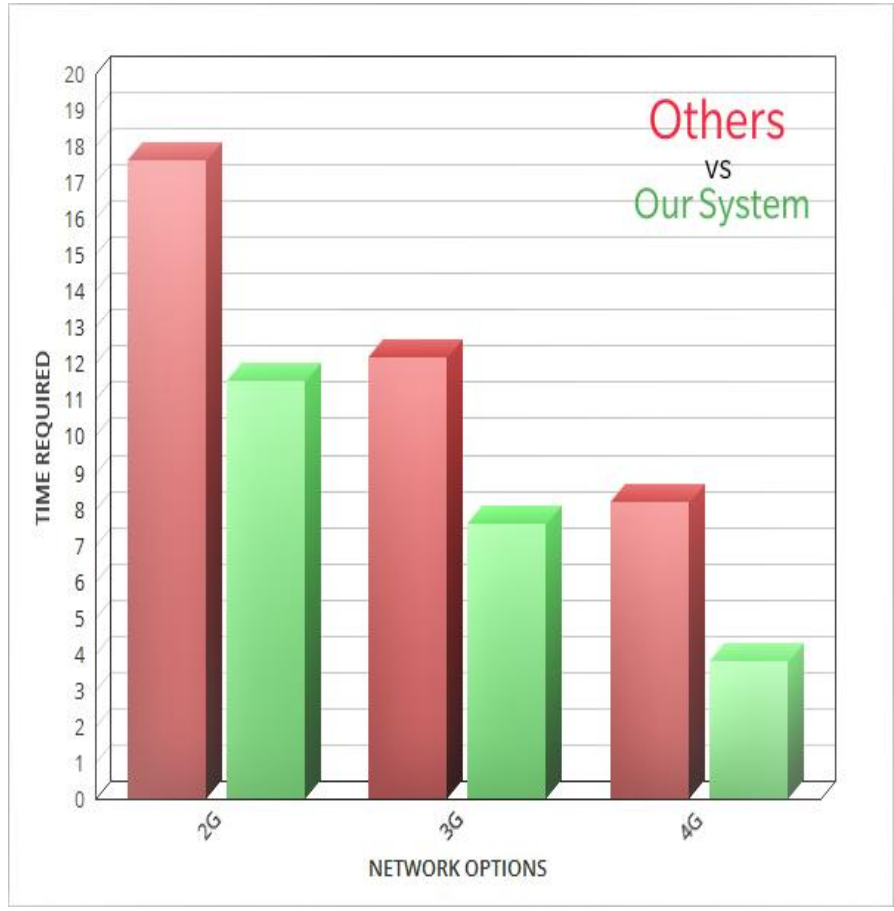

Fig. 5. Result of obstacle and collision detection

\section{REFERENCES}

[1] Blindness: vision 2020 The global initiative for the elimination of avoidable blindness. Retrieved from http://www.who.int/mediacentre/factsheets/fs213/en/

[2] Visual impairment and blindness. Retrieved from http://www.who.int/mediacentre/factsheets/fs282/en/

[3] A. Iqbal, U. Farooq, H. Mahmood, and M.U. Asad, "A low cost artificial vision system for visually impaired people," 2009 Second International Conference on Computer and Electrical Engineering, pp. 474-479, December 2009.

[4] Sandesh Chinchole, Samir Patel, "Artificial Intelligence and Sensor Based Assistive System for Visually ImpairedPeople", Proceedings of the International Conference onIntelligent Sustainable Systems (ICISS 2017)

[5] S. Shoval, J. Borenstein, and Y . Koren, "Mobile robot obstacle avoidance in a computerized travel aid for the blind," Proceedings of the 1994 IEEE International Conference on Robotics and Automation, vol. 3, pp. 2023-2028, May 1994.

[6] Google TalkBack. Retrieved from https://en.wikipedia.org/wiki/Google_Talkback

[7] Computer Vision API version 1.0, October 2017.Retrieved from https://docs.microsoft.com/enin/azure/cognitive- services/computer-vision/home

[8] A. Linn. Teaching computers to describe images as people would, April 2016. Retrieved from https://blogs.microsoft.com/ai/2016/04/14/teachingcompute rs-to- 